Classification

Physics Abstracts

07.85.-m - 47.55.Mh - 81.70.Yp

\title{
X-ray Microradiographic Determination of Diffusion Coefficients within Porous Media Based on Fick's 2nd Law: Theory and Error Analysis
}

Stephanie E.P. Dowker $\left({ }^{1}\right)$, Paul Anderson $\left({ }^{2}\right)$ and James C. Elliott $\left({ }^{2}\right)$

$\left({ }^{1}\right)$ Department of Conservative Dentistry, Faculty of Clinical Dentistry, Queen Mary and Westfield College, Turner Street, London E1 2AD, U.K.

$\left({ }^{2}\right)$ Department of Biophysics in Relation to Dentistry, Faculty of Clinical Dentistry, Queen Mary and Westfield College, Turner Street, London E1 2AD, U.K.

(Received December 15, 1995; accepted January 5, 1996)

\begin{abstract}
A method based on Fick's 2nd Law is described for the determination of local effective diffusion coefficients, within porous media, from X-ray absorption measurements. Numerical methods were used to simulate concentrations and corresponding X-ray absorption data during diffusion of an X-ray absorbing species within the fluid phase. Errors arising from X-ray counting statistics were investigated analytically and by Monte Carlo simulations. The influence of experimental conditions on errors is discussed.
\end{abstract}

\section{Nomenclature}

$a \quad$ defined in equation (18)

$A \quad$ length of solid in $x$-direction [cm]

$b$ defined in equation (18)

$B$ constant thickness of solid in $z$-direction [cm]

$c$ dimensionless variable, $C / C_{0}$

$C$ concentration of diffusing species within fluid $\left[\mathrm{g} \mathrm{cm}^{-3}\right]$

$C_{0} \quad$ constant concentration at $x=0, t>0\left[\mathrm{~g} \mathrm{~cm}^{-3}\right]$

$D^{*} \quad$ effective diffusion tensor $\left[\mathrm{cm}^{2} \mathrm{~s}^{-1}\right]$

$D^{*} \quad$ diffusion coefficient within porous medium $\left[\mathrm{cm}^{2} \mathrm{~s}^{-1}\right]$

$D_{\mathrm{f}}$ diffusion coefficient within fluid phase $\left[\mathrm{cm}^{2} \mathrm{~s}^{-1}\right]$

F flux of diffusing species per unit area of fluid phase $\left[\mathrm{g} \mathrm{cm}^{-2} \mathrm{~s}^{-1}\right]$

$I \quad$ transmitted X-ray intensity $\left[\mathrm{s}^{-1}\right]$

$I_{0}^{*} \quad$ incident X-ray intensity $\left[\mathrm{s}^{-1}\right]$

$I_{0} \quad$ defined in equation (11) $\left[\mathrm{s}^{-1}\right]$

$m$ projected mass $\left[\mathrm{g} \mathrm{cm}^{-2}\right]$ 


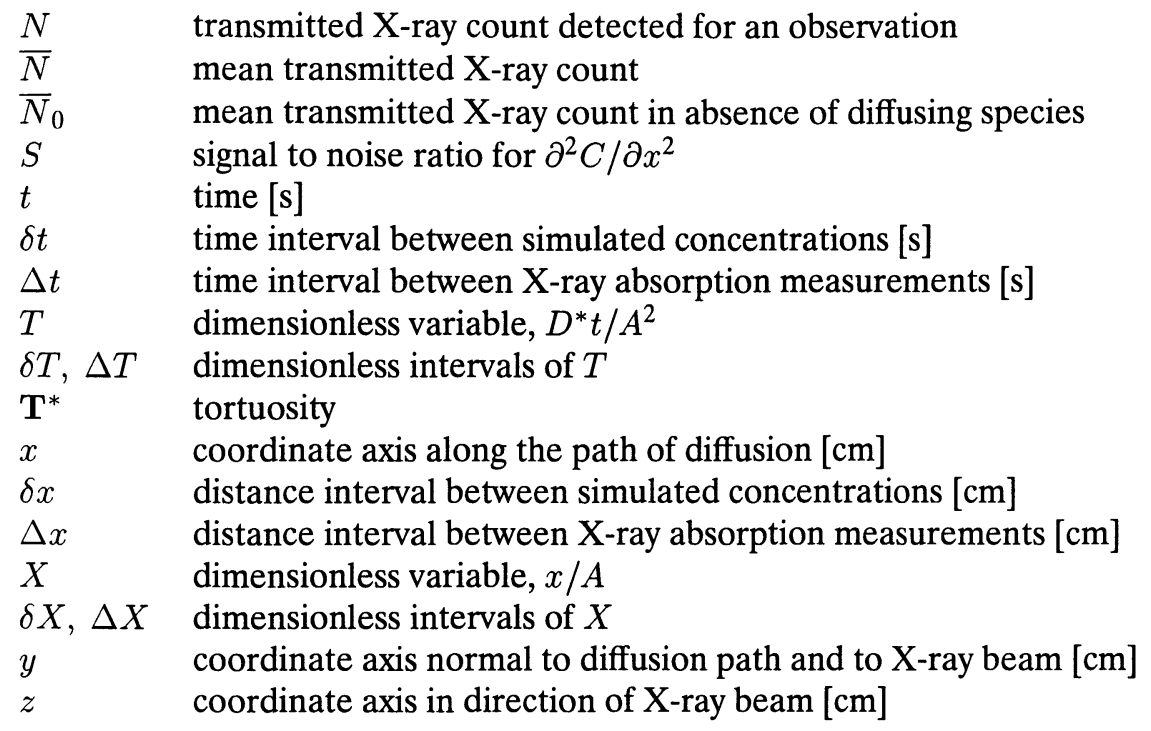

Greek Symbols

$\varepsilon \quad$ fractional volume porosity

$\mu \quad$ mass absorption coefficient $\left[\mathrm{cm}^{2} \mathrm{~g}^{-1}\right]$

$\sigma($ ) signifies standard deviation of variable within brackets

$\tau \quad$ period of concentration square wave at $x=0$ [s]

\section{SUBSCRIPTS}
d diffusing species
$\ell \quad$ liquid solvent for diffusing species
s solid

\section{Introduction}

There is no particular difficulty in determining the gross diffusional properties of porous media if they are homogeneous on a macroscopic scale. However, if these vary from place to place, there are considerable problems because local measurements within the medium are required. Our particular interest, mass transport within mineralized tissues especially with regard to caries (decay) in tooth enamel, provides a good example. This tissue is a porous medium comprising a solid phase, primarily composed of apatitic calcium phosphate crystallites, and a fluid phase composed of an aqueous solution through which there is diffusive mass transport. Dental enamel is anisotropic, since the crystallites have a needle or plate-like morphology (cross-sectional width about $30 \mathrm{~nm}$ and length $0.1-5 \mu \mathrm{m}$ or greater) and are aligned in enamel prisms approximately normal to the natural surface of the tooth. In the caries process, mineral of the solid phase dissolves, particularly below the surface or within the depth of the enamel, but may also be reprecipitated, so that the microscopic geometrical characteristics of the porous medium change within the carious lesion. Observations by polarised light microscopy show that, at this scale, porosity and pore accessibility vary gradually with the depth from the surface within a carious lesion, but are approximately constant in sound enamel [1]. In order to develop quantitative models for the reaction and transport processes in dental caries, measurements of the parameters that control the variation in mass diffusion with position, on such a scale, are required. 
The purpose of the present work was to study the theory and error analysis for determination of effective diffusion coefficients within porous media by a method that uses X-ray absorption measurements. The scale on which the effective diffusion coefficient is to be determined is very large relative to that at which microscopic pore characteristics are described, but small relative to that at which variation in macroscopic porosity becomes important.

In order to determine such local values of the effective diffusion coefficient, time-series of local concentration measurements during diffusion are required. Non-destructive methods by which series of concentration-distance profiles have been measured for 1-dimensional diffusion include: optical absorption spectrometry [2,3] and X-ray projection microscopy [4] for liquid media; synchrotron X-ray fluorescence microprobe analysis for gels [5]; and scanning neutron radiography [6] for porous solids. The use of 1-, 2- and 3-dimensional images acquired by NMR in the study of transport in porous materials has been reviewed by Gladden [7]. Kärger and Ruthven [8] have given a general review of methods applied to measurement of diffusion in porous solids, including optical techniques, X-ray absorption methods, NMR and ESR spin mapping.

$\mathrm{X}$-ray absorption methods have potentially wide applicability since the only essential requirement is that the diffusing species has a substantially higher X-ray absorption cross-section than other components of the system. Further, the precision with which the concentration of the diffusing species can be determined is known, because it depends on photon counting statistics. For studies of diffusion in thin sections, microradiography, particularly scanning microradiography (SMR), can be used, whilst for 3-dimensional measurements, microtomography is required [9].

In SMR, a thin sample of the porous material is stepped past a fine $\mathrm{X}$-ray beam and the transmitted photons counted for known positions in the sample (current spatial resolution $\sim 10 \mu \mathrm{m}$ ) after known time intervals during diffusion. With application of Fick's 1st and 2nd Laws, the technique has been used to determine the effective diffusion coefficient for 1-dimensional diffusion, $D^{*}$, assumed to be macroscopically homogeneous, in a fluid-filled glass frit [10].

For the method using Fick's 1st Law, the influence of experimental variables on the errors in local values of $D^{*}$ have been studied in detail [11]. A major practical disadvantage of this approach is the need for measurements to be made along the entire length of the solid beyond the position at which $D^{*}$ is to be determined. Further, application of Fick's 1 st Law to determination of local values of $D^{*}$ is inherently limited to 1-dimensional diffusion and 3-dimensional diffusion with spherical symmetry [11].

This paper describes an application of Fick's 2nd Law, which has the advantage of requiring measurements at only three positions (minimum) in the region of interest. In principle, the method is applicable to 1-, 2- and 3-dimensional diffusion.

\section{Determination of Diffusion Coefficients: Theory}

2.1 An Application of Fick's 2ND Law to Measurement of Diffusion By X-Ray ABSORPtion. - Consider a porous medium in which the solid matrix and the void space are both multiply connected. The void space is filled with a single fluid phase, within which a single component diffuses without sources or sinks. The solid matrix is rigid and invariant with time i.e. chemically inert with respect to the fluid phase. There is no mass transfer across the solid-fluid interface and no adsorption of the diffusing component of the fluid phase onto the surface of the solid.

A detailed derivation of the continuum model of a porous medium and macroscopic description of mass diffusion by volume averaging has been given by Bear and Bachmat [12], who introduce the concept of a Representative Elementary Volume (REV). In the system considered here, the medium is, in the general case, macroscopically heterogeneous with respect to geometrical 
characteristics. However it is assumed that there exists a range of REVs within which these characteristics are essentially constant. Further it is assumed that these REVs share a common range of volumes for REVs associated with the relevant state variables for diffusion.

Within a REV, a second order diffusion tensor, $\mathbf{D}^{*}$, can be defined through Fick's first law

$$
\mathbf{F}=-\mathbf{D}^{*} \nabla C
$$

where $\mathbf{F}$ is the flux per unit area of the fluid phase and $C$ is the concentration of diffusant within the fluid, both averaged over the REV.

From this, Fick's second law is

$$
\frac{\partial C}{\partial t}=\nabla \cdot\left(\mathbf{D}^{*} \nabla C\right)
$$

where $t$ is time. The link to the microscopic scale is given by

$$
\mathbf{D}^{*}=D_{\mathrm{f}} \mathbf{T}^{*}
$$

where $\mathbf{T}^{*}$ is a second order tortuosity tensor, as defined by Bear and Bachmat [12]. $D_{\mathrm{f}}$, the molecular diffusion coefficient within the fluid, may vary with concentration, but is assumed to be constant over the small range of concentrations used to measure $\mathbf{D}^{*}$ (see later). From equations (2) and (3),

$$
\frac{\partial C}{\partial t}=D_{\mathrm{f}} \nabla \cdot\left(\mathbf{T}^{*} \nabla C\right)
$$

In general, if measurements of $C$ as a function of time are made in 3-dimensions in voxels surrounding a point for several directions of flux, it will be possible in principle to determine the 9 elements of the diffusion tensor $\mathbf{D}^{*}$ at the point, and hence $\mathbf{T}^{*}$ if $D_{\mathrm{f}}$ is known for the particular concentration of diffusant. However, as discussed by Crank [13], if measurements are restricted to 1-dimension (taken as the $x$ direction), the one-dimensional diffusion equation

$$
\frac{\partial C}{\partial t}=D^{*} \frac{\partial^{2} C}{\partial x^{2}}
$$

with $D^{*}$ as a 1-dimensional effective diffusion coefficient is only valid if the flow is restricted to the $x$ direction. In this case, $D^{*}=D_{11}^{*}$. If $x$ is also parallel to one of the principal axes of the diffusion tensor, $D^{*}$ will be equal to $D_{1}$, one of the three principal diffusion coefficients. For an anisotropic solid and one set of 1-dimensional measurements, it will not be possible to ensure that equation (5) is valid, unless there is symmetry which determines the orientation of a principal axis. In this case, the experiment can be set up so that the principal axis is parallel to the direction of flow. Dental enamel is likely to be an example of such a system, because it comprises an approximately parallel arrangement of prisms (as described earlier) whose direction probably coincides with a principal axis of the diffusion tensor.

Assuming that equation (5) is valid, we consider one-dimensional diffusion in the $x$-direction in a parallelepiped of porous solid of length $A$ and depth $B$ (Fig. 1). This might be a physical slab whose diffusant concentrations can be determined by direct radiographic methods, or a row of pixels in the middle of a larger block of porous solid in which the diffusant concentration is determined by X-ray microtomography. Consider a general distance $p \Delta x$ and general time $q \Delta t$, where $p$ and $q$ are integers, and $\Delta x$ and $\Delta t$ are respectively the distance and time intervals between measurements (Fig. 2). The problem is to sample points around $(p, q)$ in order to determine the value of $D^{*}$ at $p$. The values of $\partial C / \partial t$ and $\partial^{2} C / \partial x^{2}$ can be estimated as:

$$
\begin{aligned}
\frac{\partial C}{\partial t} & \approx \frac{C_{p, q+1}-C_{p, q}}{\Delta t} \\
\frac{\partial^{2} C}{\partial x^{2}} & \approx \frac{C_{p+1, q}-2 C_{p, q}+C_{p-1, q}}{(\Delta x)^{2}}
\end{aligned}
$$




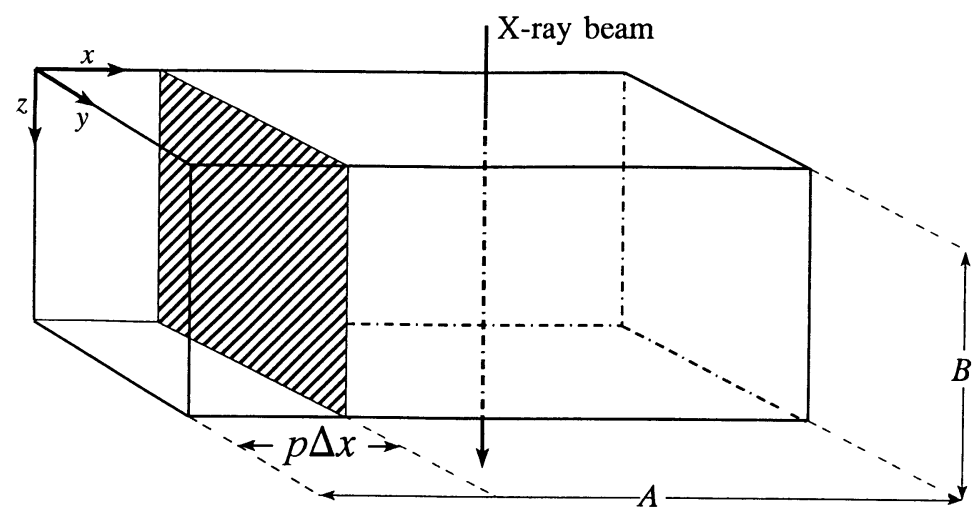

Fig. 1. - Schematic of the dimensions and coordinate system for one-dimensional diffusion in a porous solid.

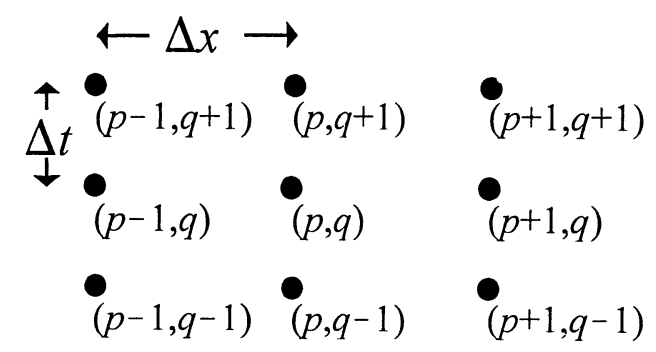

Fig. 2. - Grid of data points used in calculations.

However the result from equation (7) refers to the time $q \Delta t$, whereas the value of $\partial C / \partial t$ estimated by equation (6) refers to a time between $q \Delta t$ and $(q+1) \Delta t$. A closer approximation of $\partial^{2} C / \partial x^{2}$ at this latter time is given by:

$$
\frac{\partial^{2} C}{\partial x^{2}} \approx \frac{C_{p+1, q+1}-2 C_{p, q+1}+C_{p-1, q+1}+C_{p+1, q}-2 C_{p, q}+C_{p-1, q}}{2(\Delta x)^{2}}
$$

An alternative approach, which avoids correlation between the estimates of $\partial C / \partial t$ and $\partial^{2} C / \partial x^{2}$, is to determine $\partial C / \partial t$ from:

$$
\frac{\partial C}{\partial t} \approx \frac{C_{p, q+1}-C_{p, q-1}}{2 \Delta t}
$$

and $\partial^{2} C / \partial x^{2}$ from equation (7).

In the following Section, we discuss the measurement of concentrations by X-ray absorption. In order that the diffusion process, as characterized by such measurements within this system, can be described by a continuous model, the X-ray beam diameter (and for measurements derived from microtomography measurements, the voxel size) must be within upper and lower limits determined by the requirement that the volume of the medium over which averaging is applied is within the relevant range of REVs, so that the measurement can be referred to a point. 
2.2 Measurement of Concentrations by X-Ray Absorption. - As discussed earlier, concentrations can either be measured by microradiographic or microtomographic methods. The analysis in the rest of the paper will assume measurements from radiography (Fig. 1). A similar analysis could be derived for microtomography, except that Figure 1 would be replaced by a block of pixels within the solid, in which the linear absorption coefficient projection was given by the reconstruction algorithm and with a standard deviation depending differently on the incident $\mathrm{X}$ ray intensity (see later).

For a beam of monochromatic X-ray radiation in the $z$-direction (Fig. 1), absorption due to each of the components of the sample is independent.

$$
I=I_{0}^{*} \exp \left(-\mu_{\mathrm{s}} m_{\mathrm{s}}-\mu_{\ell} m_{\ell}-\mu_{\mathrm{d}} m_{\mathrm{d}}\right)
$$

where $I_{0}^{*}$ (assumed to be constant over the times and distances for the measurements, and to be measurable without photon statistical error) and $I$ are respectively the incident and transmitted intensities, and $\mu$ and $m$ are respectively the mass absorption coefficient and projected mass for the solid (s), liquid solvent in the pores $(\ell)$ and the diffusing species (d).

It is assumed that during diffusion the increased concentration of the diffusing species within the pores produces a negligible reduction in the projected mass of the solvent. Thus to a very good approximation, absorption due to the solvent is constant. Defining $I_{0}$ by:

$$
I_{0}=I_{0}^{*} \exp \left(-\mu_{\mathrm{s}} m_{\mathrm{s}}-\mu_{\ell} m_{\ell}\right)
$$

absorption by the diffusing species can be considered independently:

$$
I=I_{0} \exp \left(-\mu_{\mathrm{d}} m_{\mathrm{d}}\right)
$$

Substituting $\varepsilon B C=m_{\mathrm{d}}$, where $\varepsilon$ is porosity, so that over the REV the mean X-ray pathlength through the pores is $\varepsilon B$ :

$$
C_{p, q}=\frac{1}{\mu_{\mathrm{d}} \varepsilon B}\left(\ln I_{0}-\ln I_{p, q}\right)
$$

If the X-ray intensity $I_{p, q}$ is measured by a photon counting system, the number of photons, $N_{p, q}$, detected in a fixed time, has a Poisson distribution with standard deviation $\bar{N}_{p, q}^{1 / 2}$, where $\bar{N}_{p, q}$ is the mean number of photons detected in the fixed time. Thus the fractional error in $I_{p, q}$ is:

$$
\frac{\sigma\left(I_{p, q}\right)}{I_{p, q}}=\frac{\sigma\left(\bar{N}_{p, q}\right)}{\bar{N}_{p, q}}=\frac{1}{\sqrt{\bar{N}_{p, q}}}
$$

where $\sigma($ ) indicates the standard deviation of the respective variable within the brackets.

2.3 Determination of Diffusion Coefficients from X-Ray Absorption MeasureMENTS. - The concentrations in equations (6) - (9) can be expressed in terms of X-ray absorption measurements using equation (13). Three methods of estimating $D^{*}$ from equation (5) are considered.

Method A, utilizing equations (6) and (7), requires four measurements giving:

$$
D_{p, q}^{*}=\frac{(\Delta x)^{2}}{\Delta t}\left(\frac{\ln I_{p, q+1}-\ln I_{p, q}}{\ln I_{p+1, q}-2 \ln I_{p, q}+\ln I_{p-1, q}}\right)
$$

Method B, utilizing equations (9) and (7), requires five measurements giving:

$$
D_{p, q}^{*}=\frac{(\Delta x)^{2}}{2 \Delta t}\left(\frac{\ln I_{p, q+1}-\ln I_{p, q-1}}{\ln I_{p+1, q}-2 \ln I_{p, q}+\ln I_{p-1, q}}\right)
$$


and Method C, utilizing equations (6) and (8), requires six measurements giving :

$$
D_{p, q}^{*}=\frac{2(\Delta x)^{2}\left(\ln I_{p, q+1}-\ln I_{p, q}\right)}{\Delta t\left(\ln I_{p+1, q+1}-2 \ln I_{p, q+1}+\ln I_{p-1, q+1}+\ln I_{p+1, q}-2 \ln I_{p, q}+\ln I_{p-1, q}\right)}
$$

Note that these equations do not require knowledge of the local porosity or of the mass absorption coefficient for any component.

\section{Numerical Simulation of Concentrations during Diffusion}

The method for determination of $D^{*}$ requires only that $D^{*}$ and $\varepsilon$ are constant over the measurement distance i.e. only between three measurements. However, for simplicity, a single set of concentrations simulating diffusion with $D^{*}$ independent of distance was calculated. Subsets of concentrations at three adjacent positions were then drawn from it for separate consideration.

Using the dimensionless variables:

$$
X=x / A, \quad T=D^{*} t / A^{2}, \quad c=C / C_{0}
$$

where $C_{0}$ is a constant concentration, a set of dimensionless concentration data was calculated using the explicit finite difference method [13], using intervals $\delta X=1 \times 10^{-3}$ and $\delta T=4 \times$ $10^{-7}$ which conform to the constraint for a stable solution [14]. Using the values of $D^{*}$ and $A$ in Table I, $\delta X$ and $\delta T$ correspond to a distance interval, $\delta x=1 \times 10^{-3} \mathrm{~cm}$, and a time interval, $\delta t=4 \times 10^{-2} \mathrm{~s}$. From this set of concentrations, subsets corresponding to the very much larger experimental intervals of distance $\Delta x$, typically $0.04 \mathrm{~cm}$, and time $\Delta t$, typically $500 \mathrm{~s}$, were selected. By the choice of $\delta x \ll \Delta x$ and $\delta t \ll \Delta t$, the errors resulting from truncation of the Taylor series expansion in the explicit finite difference calculation could be neglected.

Table I. - Experimental variables used in simulation.

\begin{tabular}{|l|l|}
\hline \multicolumn{2}{|c|}{ Porous solid } \\
\hline $\begin{array}{l}\text { Length in direction of diffusion, } A \\
\text { Thickness in direction of X-ray beam, } B \\
\text { Porosity, } \varepsilon\end{array}$ & $1.0 \mathrm{~cm}$ \\
\hline \multicolumn{2}{|c|}{ Diffusing species $(\mathrm{KI})$} \\
\hline \multicolumn{2}{|c|}{0.25} \\
\hline Effective diffusion coefficient, $D^{*}$ & $1.0 \times 10^{-5} \mathrm{~cm}^{2} \mathrm{~s}^{-1}$ \\
Relative molecular mass & 166 \\
Mass absorption coefficient for MoK &,$\mu_{\mathrm{d}}$ \\
\hline \multicolumn{2}{|c|}{$32.08 \mathrm{~g}^{-1} \mathrm{~cm}^{2}$} \\
\hline Concentration in fluid phase at $x=0, C_{0}$ & $1.0 \mathrm{~mol} \mathrm{dm}{ }^{-3}$ \\
Measurement distance interval, $\Delta x$ & $0.04 \mathrm{~cm}$ \\
Measurement time interval, $\Delta t$ & $500 \mathrm{~s}$ \\
\hline
\end{tabular}




\section{Assessment of Errors in Diffusion Coefficients}

4.1 Errors Due to Sampling Using Finite Distance and Time Intervals. - The use of data at finite distance and time intervals in equations (6) - (9) results in approximation of the continuous functions in equation (5), so that each calculated effective diffusion coefficient differs from $D^{*}$. These systematic errors, which limit the accuracy with which $D^{*}$ can be determined, were investigated using simulated dimensionless concentration data with sampling intervals $\Delta X \gg \delta X$ and $\Delta T \gg \delta T$.

Contour lines of constant fractional error in $D^{*}$ are shown on an $X: T$ grid (Fig. 3). Method A resulted in large errors over a wide range of $X$ and $T$ (Figs. 3a,d). By contrast, the use of Methods $B$ and $C$ provided extensive regions within which the fractional error was $<|0.02|$, for the four conditions investigated (Figs. 3b,c,e,f).

Thus a range of experimental conditions corresponding to such combinations of $\Delta X$ and $\Delta T$ could be used with Methods B or C, without producing substantial errors due to finite sampling. Small values of $T$, at which large errors arise, must be avoided.

\subsection{Errors Arising from Photon Counting Statistics.}

4.2.1 Ranges of Simulated Conditions. - We now consider how the precision with which $D_{p, q}^{*}$ can be determined depends on counting statistics. The results of a Monte Carlo method are compared with analytical estimates of the errors in $D_{p, q}^{*}$. Simulated experimental conditions were chosen for which the fractional error due to finite sampling (Sect. 4.1) would be negligible, in comparison with the error due to photon counting statistics for the range of $\bar{N}_{0}$ relevant to most practical applications. For Methods B and C, error determination was restricted to ranges of distance $(0.12$ to $0.84 \mathrm{~cm})$ and time (3000 to $8000 \mathrm{~s})$ : the fractional error due to finite sampling, generally $<|0.02|$ (Figs. 3b,c), was neglected. Method A was not considered, because of its large errors.

4.2.2 Monte Carlo Method. - The dimensioned concentration data (Sect. 3), with $\mu_{\mathrm{d}}$ as in Table I, was used to provide a range of count measurements required for the calculations by Method B, equation (16), and Method C, equation (17). For the conditions studied (Sect. 4.2.1), the variation in $\bar{N}_{p}$ with time over $500 \mathrm{~s}$ was very close to linear, so that the effect of finite counting time could be neglected.

The dimensioned data were first transformed to produce count data corresponding to mean count values for infinite counting time, $\bar{N}_{p, q}$. Simulated experimental count data were then calculated for each value of $\bar{N}_{p, q}$, by use of a look-up table for 10000 values of the cumulative distribution function (CDF) of a Gaussian distribution. (For these large values of $\bar{N}$, the Gaussian distribution provides a very good approximation to the Poisson distribution.) A pseudo-random generator between 1 and 10000 was used to locate a point on the CDF curve, and the CDF value multiplied by $\bar{N}_{p, q}$ to give the simulated count $N_{p, q}$. For each value of $\bar{N}_{0}$ investigated, 4000 sets of simulated data were used to investigate the distribution of $D_{p, q}^{*}$. 


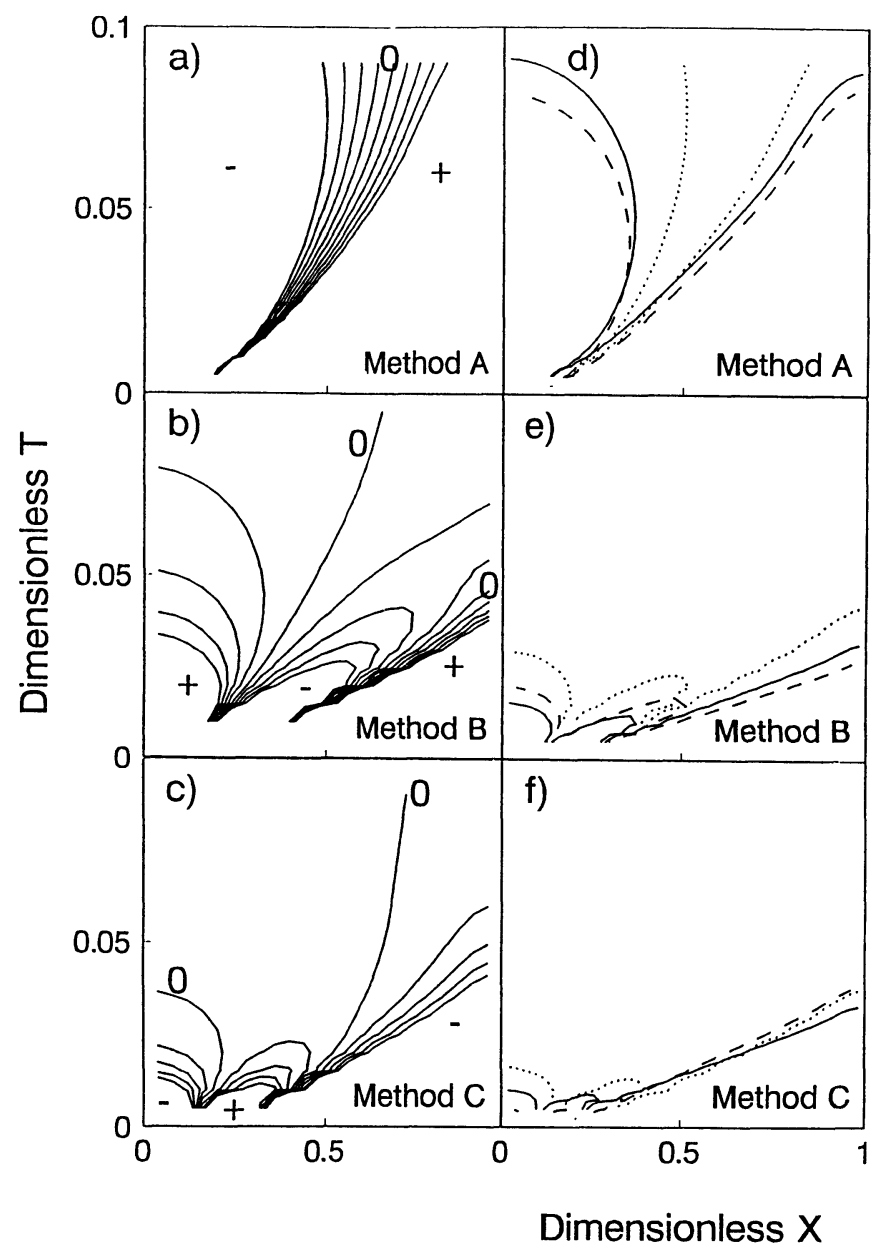

Fig. 3. - Contour lines of constant fractional error in $D^{*}$ as a function of $X$ and $T$ for $D^{*}=1.0 \times 10^{-5}$ $\mathrm{cm}^{2} \mathrm{~s}^{-1}$. Panels $\mathrm{a}, \mathrm{b}$ and $\mathrm{c}$ : contour lines at 0.005 intervals, with areas in which fractional error $>|0.02|$ marked + or - , for $\Delta X=0.04, \Delta T=0.0050$. Panels d, e and $\mathrm{f}$ : contour lines of fractional error $=|0.02|$ for $\Delta X=0.02, \Delta T=0.0025(-), \Delta X=0.04, \Delta T=0.0025(--)$, and $\Delta X=0.02, \Delta T=0.0050$ $(\ldots)$.

4.2.3 Analytical Estimation. - The error in $D_{p, q}^{*}$ was estimated by combination of errors (taken as the standard deviations from Eq. (14)) in the intensities in equations (16) and (17), using the rules based on partial differentiation. Equations (16) and (17) were rewritten as:

$$
D_{p, q}^{*}=\frac{k(\Delta x)^{2}}{\Delta t} \frac{a}{b}
$$

where $a$ and $b$ are respectively the logarithmic numerator and denominator functions from either equation and $k$ is a constant. 
For Method B:

$$
\begin{aligned}
\frac{\sigma(a)}{a} & =\frac{\left(\frac{1}{N_{p, q+1}}+\frac{1}{N_{p, q-1}}\right)^{1 / 2}}{\ln \frac{N_{p, q}}{N_{p, q+1}}} \\
\frac{\sigma(b)}{b} & =\frac{\left(\frac{1}{N_{p+1, q}}+\frac{2}{N_{p, q}}+\frac{1}{N_{p-1, q}}\right)^{1 / 2}}{\ln \frac{N_{p, q}^{2}}{N_{p+1, q} N_{p-1, q}}}
\end{aligned}
$$

For Method C:

$$
\begin{aligned}
\frac{\sigma(a)}{a} & =\frac{\left(\frac{1}{N_{p, q+1}}+\frac{1}{N_{p, q}}\right)^{1 / 2}}{\ln \frac{N_{p, q+1}}{N_{p, q}}} \\
\frac{\sigma(b)}{b} & =\frac{\left(\frac{1}{N_{p+1, q+1}}+\frac{2}{N_{p, q+1}}+\frac{1}{N_{p-1, q+1}}+\frac{1}{N_{p+1, q}}+\frac{2}{N_{p, q}}+\frac{1}{N_{p-1, q}}\right)^{1 / 2}}{\ln \frac{N_{p, q}^{2}}{N_{p+1, q+1} N_{p-1, q+1} N_{p+1, q} N_{p-1, q}}}
\end{aligned}
$$

$\sigma\left(D_{p, q}^{*}\right)$ is given by:

$$
\left(\frac{\sigma\left(D_{p, q}^{*}\right)}{D_{p, q}^{*}}\right)^{2}=\left(\frac{\sigma(a)}{a}\right)^{2}+\left(\frac{\sigma(b)}{b}\right)^{2}+\frac{2 \operatorname{cov}(a, b)}{a b}
$$

where $\operatorname{cov}(a, b)$ is the covariance of $a$ and $b$. For Method B, $\operatorname{cov}(a, b)$ is zero, because each measurement is independent. For Method C, $N_{p, q}$ and $N_{p, q+1}$ are used in the estimation of both $\partial C / \partial t$ and $\partial^{2} C / \partial x^{2}$, but as most of the count measurements used are independent, $\operatorname{cov}(a, b)$ was again set to zero.

The fractional errors in $D^{*}, a$ and $b$ were determined for Methods B and C, within the same range of experimental conditions used for the Monte Carlo simulations.

4.2.4 Results. - There was good agreement between $\sigma\left(D^{*}\right)$ determined by the analytical method and $\sigma\left(D^{*}\right)$ determined by the Monte Carlo method for $\bar{N}_{0} \geq 10^{8}$ (Fig. 4). As expected, there was an approximately $n$-fold reduction in standard deviation with $n^{2}$-fold increase in $\bar{N}_{0}$, for a given combination of position and time. At lower $\bar{N}_{0}\left(\leq 10^{8}\right), \sigma\left(D^{*}\right)$ from the Monte Carlo method deviated increasingly erratically from the corresponding values predicted analytically. Inspection of the distributions of $D^{*}$ from the Monte Carlo method showed that high $\bar{N}_{0}$ gave symmetrical distributions, but with reduced $\bar{N}_{0}$, the distributions became increasingly skewed and had more extreme outliers. This results from the dependence of the method on determination of small differences between count values and their subsequent division. Calculation of the fractional errors in the estimates of $\partial C / \partial t$ and $\partial^{2} C / \partial x^{2}$ showed that the error in $\partial^{2} C / \partial x^{2}$ predominates (Fig. 5). From the numerators of equations (16) and (17), the signal to noise ratio, $S$, for $\partial^{2} C / \partial x^{2}$, in Method B is

$$
S=\frac{-\bar{N}_{p+1, q}+2 \bar{N}_{p, q}-\bar{N}_{p-1, q}}{\left(\bar{N}_{p+1, q}+2 \bar{N}_{p, q}+\bar{N}_{p-1, q}\right)^{1 / 2}}
$$




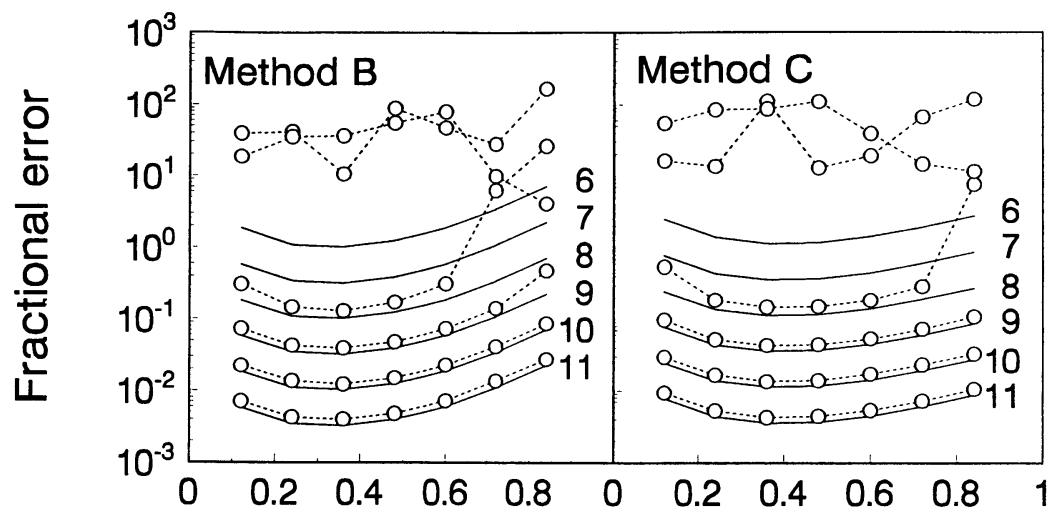

Distance / cm

Fig. 4. - Variation with distance of the fractional errors in $D^{*}$ determined analytically $(-)$ and by Monte Carlo calculations $(\bigcirc--\bigcirc)$ for $\bar{N}_{0}$ from $10^{6}$ to $10^{11}$ (lines labelled with exponent) at $5000 \mathrm{~s}$.

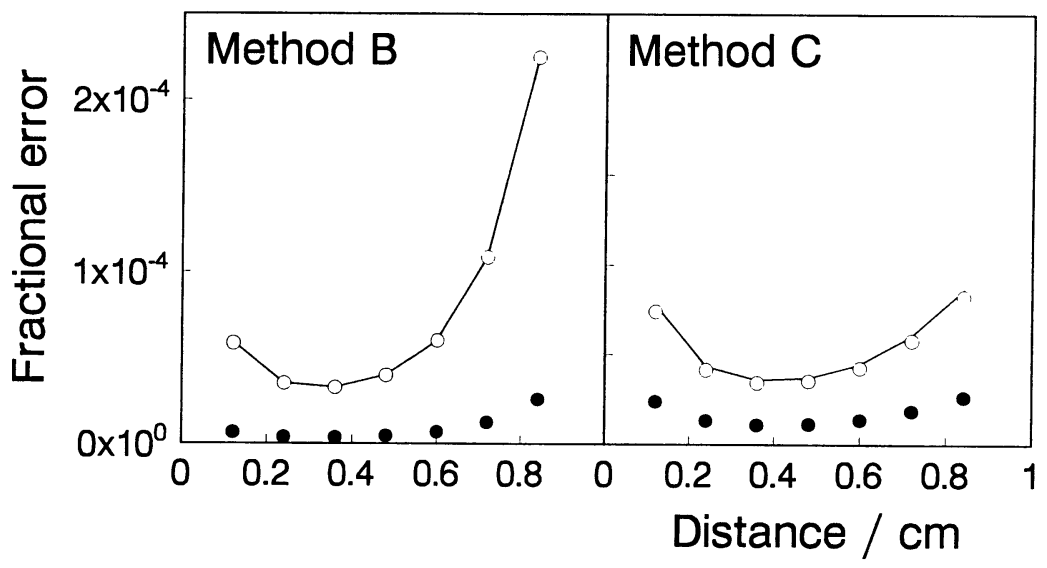

Fig. 5. - Variation with distance of fractional errors in $D^{*}(-), \partial C / \partial t(\bullet \bullet)$ and $\partial^{2} C / \partial x^{2}(\circ \bigcirc)$ determined analytically for $\bar{N}_{0}=10^{15}$ at $5000 \mathrm{~s}$.

and in Method C,

$$
S=\frac{-\bar{N}_{p+1, q+1}+2 \bar{N}_{p, q+1}-\bar{N}_{p-1, q+1}-\bar{N}_{p+1, q}+2 \bar{N}_{p, q}-\bar{N}_{p-1, q}}{\left(\bar{N}_{p+1, q+1}+2 \bar{N}_{p, q+1}+\bar{N}_{p-1, q+1}+\bar{N}_{p+1, q}+2 \bar{N}_{p, q}+\bar{N}_{p-1, q}\right)^{1 / 2}}
$$

Under the conditions investigated, for Methods B and C, there was an inverse relationship between $S$ and the observed fractional error in $D^{*}$ for $S \gtrsim 9$, which increased rapidly for $S \lesssim 9$ (Fig. 6).

In order to derive results comparable to the above for diffusion coefficients derived from linear absorption coefficients determined in microtomography experiments, the standard deviation of this coefficient needs to be known in terms of the recorded photons. This relation has been discussed by Davis [15]. 

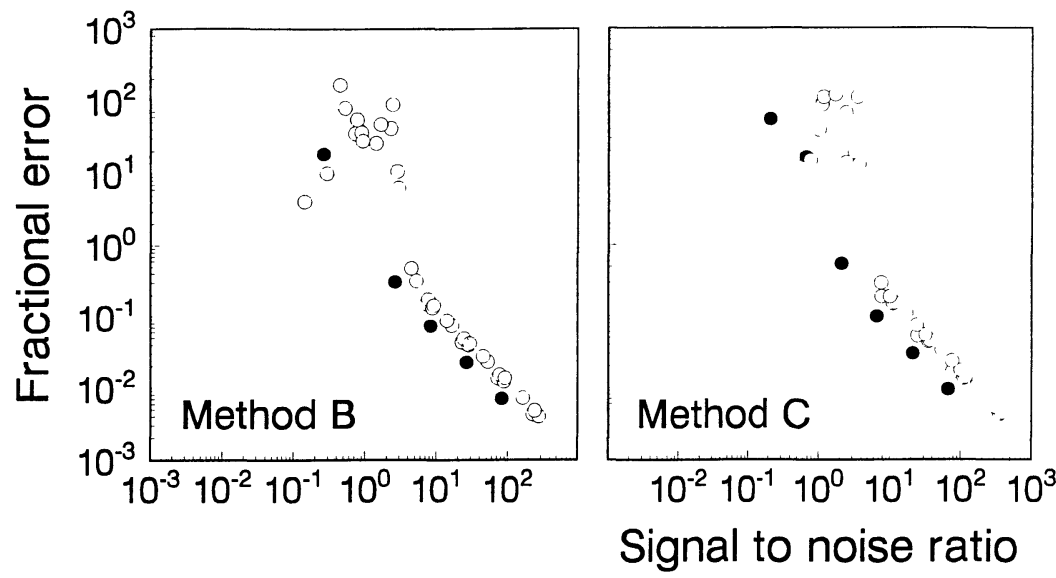

Fig. 6. - Variation in observed fractional error in $D^{*}$ (for $\bar{N}_{0}$ from $10^{6}$ to $10^{11}$ ) with signal to noise ratio, at $t=5000 \mathrm{~s}$, for $x=0.12 \mathrm{~cm}(\bullet)$ and $x=0.24,0.36, \ldots, 0.84 \mathrm{~cm}(\bigcirc)$.

The above discussion shows how the precision of $D^{*}$ can be improved by increasing the number of transmitted photons counted. However the usable flux obtainable from a laboratory X-ray source is relatively small and the potential for increasing counting time is constrained by the dynamic nature of the diffusion process. In the following section, we consider alternative strategies for reducing errors.

\section{Strategies for Reducing Errors in $D^{*}$}

$5.1 \partial C / \partial t$ And $\partial^{2} C / \partial x^{2}$ Summed Over Successive Time Intervals. - Provided that $D_{\mathrm{f}}$ is independent of concentration, $D_{p, q}^{*}$ in equations (16) and (17) is constant for all values of $q$, so that for both equations a new numerator and denominator can be derived by separate summation over $q$. For sets of measurements chosen to be independent in Method B (times $3 q, 3 q \pm 1$ ):

$$
D_{p}^{*}=\frac{(\Delta x)^{2}}{2 \Delta t}\left(\frac{\Sigma\left(\ln I_{p, 3 q+1}-\ln I_{p, 3 q-1}\right)}{\Sigma\left(\ln I_{p+1,3 q}-2 \ln I_{p, 3 q}+\ln I_{p-1,3 q}\right)}\right)
$$

and independent sets in Method C (times $2 q, 2 q+1)$ :

$$
\begin{aligned}
D_{p}^{*} & = \\
\frac{2(\Delta x)^{2}}{\Delta t} & \frac{\Sigma\left(\ln I_{p, 2 q+1}-\ln I_{p, 2 q}\right)}{\Sigma\left(\ln I_{p+1,2 q+1}-2 \ln I_{p, 2 q+1}+\ln I_{p-1,2 q+1}+\ln I_{p+1,2 q}-2 \ln I_{p, 2 q}+\ln I_{p-1,2 q}\right)}
\end{aligned}
$$

The reduction in error by use of this method is illustrated in Figure 7.

If $\varepsilon$ is constant over an extended distance, an improved estimate of $D^{*}$ can be obtained by extending the summations over $p$. Sets of measurements with a minimum spacing of $(3 p \pm 1)$ will ensure that each set is independent.

5.2 Use of Square Wave Driven Periodic Concentrations. - This approach is based on a phenomenon analogous to that described for conduction of heat in solids [16]. If a square wave function of concentration with period $\tau$ is imposed at $x=0$, the concentration oscillates 


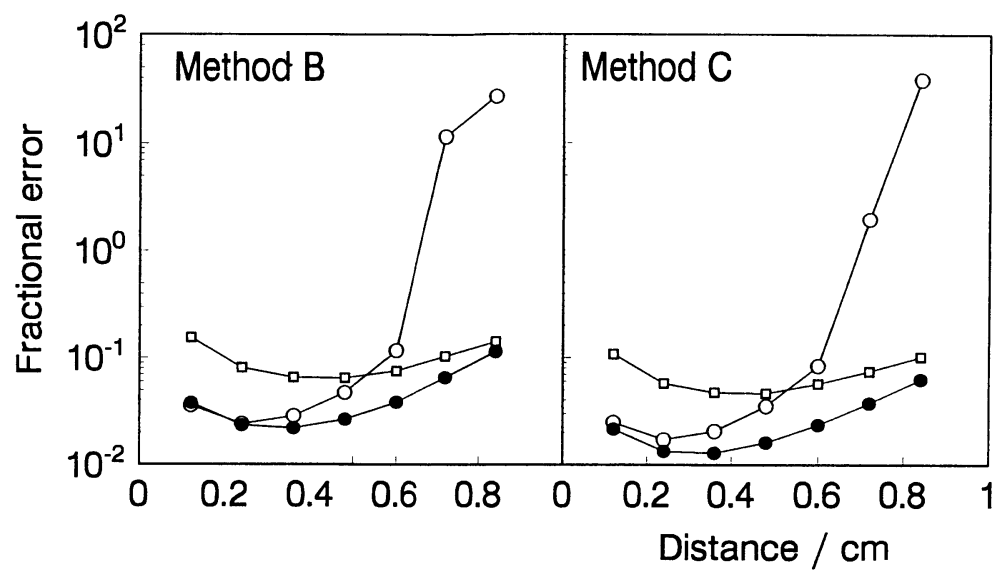

Fig. 7. - Variation in observed fractional error in $D^{*}$ with distance for $\bar{N}_{0}=10^{9}$, determined from estimates of $\partial C / \partial t$ and $\partial^{2} C / \partial x^{2}$ at $3000 \mathrm{~s}(O)$ and $8000 \mathrm{~s}(\square)$. Fractional error from $\Sigma(\partial C / \partial t)$ and $\Sigma\left(\partial^{2} C . / \partial x^{2}\right)$ summed for $3000,4000, \ldots, 8000 \mathrm{~s}(\bullet)$.

with position and time, and with increasing time, tends to a stable cycle with period $\tau$. Then, to a good approximation, a concentration at time $t$ is equal to that at $t+n \tau$, where $n$ is a positive integer. Thus if $\Delta t$ is chosen so that $\tau=k \Delta t$, where $k$ is an integer, the counts $N_{p, q}, N_{p, q+k}, \ldots$ $N_{p, q+n k}$ can be averaged. The effect of averaging over $n$ cycles is equivalent to an $n$-fold increase in $\bar{N}_{0}$ and can be determined from Figure 4.

\section{Discussion}

The determination of local values of $D^{*}$ from X-ray absorption measurements at finite distance and time intervals requires the approximation of continuous functions. For Fick's 2nd Law, the simplest of these requires only four measurements (Method A) but, for the sampling intervals of $\Delta X$ and $\Delta T$ investigated, there are large fractional errors in $D^{*}$ outside a narrow range of $X$ and $T$ (Fig. 3d). The scope for increasing the useful range of $X$ and $T$ by reducing $\Delta X$ and $\Delta T$ is constrained by the concomitant reduction in precision of measurements from counting statistics (see below). However, if five (Method B) or six (Method C) measurements are used to approximate the continuous functions, the fractional error in $D^{*}$ is $<|0.02|$ over an extensive $X: T$ region for a range of $\Delta X$ and $\Delta T$ combinations (Figs. 3e,f). Large errors are associated with very large, or very small, changes in concentration with distance or time, so measurements at these extremes cannot be used. Nevertheless, as in the application of Fick's 1st Law [11], it should generally be possible to select experimental values for $\Delta x$ and $\Delta t$ so that the errors due to finite sampling intervals will be acceptably small over a substantial period of time during diffusion.

In many cases, the dominant error originates from photon counting statistics. For a laboratory $\mathrm{X}$-ray source the usable flux is typically $10^{3}$ to $10^{4}$ photons per second for a $20 \mu \mathrm{m}$ diameter beam and energy dispersive detector set to collect the target's characteristic radiation, so optimization of experimental conditions is important.

For a given $\bar{N}_{0}$, precision in the estimate of $D^{*}$ is improved by increasing the sampling intervals, since the numerators in equations (15) - (17) increase with $\Delta t$ and the denominators increase with $\Delta x$. The optimum sampling intervals are therefore the largest for which acceptably small errors due to finite sampling (Sect. 4.1) can be obtained. 
An optimal range for the sample thickness, in order to detect concentration changes for the diffusing species, can be calculated [11]. Knowledge of the porosity of the medium and the linear absorption coefficients of the solid and solution within the range of composition during diffusion are required.

An estimate of the error arising from counting statistics is readily found by analytical methods (Sect. 4.2.3). Since the differences between $N_{p+1, q+1}, N_{p, q+1}, \ldots N_{p-1, q-1}$ are very small relative to the size of $N_{p, q}$, a reasonable approximation to this analytical estimate can be obtained by replacing each $N$-term in equations (19) - (22) with a single calculated value of $N_{p, q}$. Although the correspondence between the errors determined analytically and by Monte Carlo simulation breaks down for low $\bar{N}_{0}$ (Fig. 4), the analytical estimate can be used as an indicator of the extent by which the counts must be increased to obtain a usable precision. The reduction in error can be estimated for each of the strategies described in Section 5. Thus it is normally possible to determine whether an acceptable precision can be obtained by summation within a single infusion or effusion experiment (Sect. 5.1) or whether the experiment must be modified so that measurements of steady periodic concentrations can be used (Sect. 5.2). When the experimental measurements have been made, the signal to noise ratio for the overall estimate of $\partial^{2} C / \partial x^{2}$ can be used to confirm the confidence interval for the determination of $D^{*}$ (Sect. 4.2.4).

In single counter scanning systems, the required mean count, $\bar{N}_{0}$, to obtain a given precision in the estimate of $D^{*}$, increases in the sequence: Method B, Method C, Fick's 1st Law Method [11]. However, for a given precision, the total required count summed over all measurements is the same order of magnitude for all three methods. Methods B and $\mathrm{C}$ respectively require only five or six separate measurements of $N$, but in the method using Fick's 1st Law [11], measurements of $N$ are required at many positions in order to estimate flux, in addition to the two measurements required for the estimation of $\partial C / \partial x$.

In the Fick's 1st Law method, the flux at a given position is determined from the change in projected mass of the diffusing species along the entire diffusion path beyond that position. Since any loss of diffusing species invalidates the method, the sample must be bounded by an impermeable plane. However this restriction does not apply for the methods using Fick's 2nd Law, since only local measurements within an REV are used.

This work and a previous experimental study [10] have shown that $D^{*}$ can be determined locally within a porous solid from X-ray absorption measurements using Fick's 2 nd Law. The main discussion has been with reference to 1-dimensional diffusion, but as indicated earlier, this can be generalized to 2- or 3-dimensions. Further, X-ray methods can be extended to simultaneous diffusion of several species: elemental discrimination can be achieved by the analysis of absorption at different energies [17], intensity changes across absorption edges [18], or by X-ray fluorescence microprobe analysis using synchrotron radiation [5].

Since the determination of $D^{*}$ by quantitative neutron radiography [6] is analogous to X-ray methods, the above analysis of errors will be directly applicable.

\section{Acknowledgements}

This work was supported in part by the Special Trustees of The Royal London Hospital and presently by the Medical Research Council Grant No. G9505593-MA. Related experimental work is supported by the Central Research Fund of the University of London. 


\section{References}

[1] Darling A.I., Mortimer K.V., Poole D.F.G. and Ollis W.D., Arch. Oral. Biol. 5 (1961) 251.

[2] Tanigaki M., Kondo K., Harada M. and Eguchi W., J. Phys. Chem. 87 (1983) 586.

[3] Eguchi W., Harada M., Adachi M., Tanigaki M. and Kondo K., J. Chem. Eng. Jpn 17 (1984) 472.

[4] Rondot S., Cazaux J., Aaboubi O., Chopart J.P. and Olivier A., Science 263 (1994) 1739.

[5] Anderson P., Elliott J.C., Thomas C.R. and Van Langevelde F., X-ray Spectrometry 22 (1993) 265.

[6] Pel L., Ketelaars A.A.J., Adan O.C.G. and Van Well A.A., Int. J. Heat Mass Transfer 36 (1993) 1261.

[7] Gladden L.F., Chem. Eng. Sci. 49 (1994) 3339.

[8] Kärger J. and Ruthven D.M., Diffusion in Zeolites and other Microporous Solids (John Wiley \& Sons, New York, 1992).

[9] Elliott J.C., Anderson P., Gao X.J., Wong F.S.L., Davis G.R. and Dowker S.E.P., J. X-ray Sci. Technol. 4 (1994) 102.

[10] Anderson P., Dowker S.E.P., Elliott J.C. and Dibdin G.H., in X-ray microsc. III, A.G. Michette, G.R. Morrison and C. J. Buckley Eds. (Springer-Verlag, Berlin, Heidelberg) Springer Series Opt. Sci. 67 (1992) 435.

[11] Dowker S.E.P., Anderson P. and Elliott J.C., Microsc. Microanal. Microstruct. 6 (1995) 187.

[12] Bear J. and Bachmat Y., Introduction to Modeling of Transport Phenomena in Porous Media, Theories and Applications of Transport in Porous Media, Vol. 4 (Kluwer Academic Publishers, Dordrecht, The Netherlands, 1990).

[13] Crank J., The Mathematics of Diffusion, 2nd edn. (Oxford University Press, Oxford, 1975).

[14] Smith G.D., Numerical Solution of Partial Differential Equations (Oxford University Press, Oxford, 1965).

[15] Davis G.R., J. X-ray Sci. Technology 4 (1994) 191.

[16] Carlslaw H.S. and Jaeger J.C., Conduction of Heat in Solids, 2nd edn. (Clarendon Press, Oxford, 1959).

[17] Cazaux J., Microsc. Microanal. Microstruct. 4 (1993) 513.

[18] Anderson P., Davis G.R. and Elliott J.C., Microsc. Analysis (March 1994) 31. 